\title{
Mechanical Properties and Morphological Characterization of PLA/Chitosan/Epoxidized Natural Rubber Composites
}

\author{
Zainoha Zakaria, ${ }^{1}$ Md. Saiful Islam, ${ }^{2}$ Azman Hassan, ${ }^{3}$ M. K. Mohamad Haafiz, ${ }^{3,4}$ \\ Reza Arjmandi, ${ }^{3}$ I. M. Inuwa, ${ }^{3}$ and M. Hasan ${ }^{3}$ \\ ${ }^{1}$ Department of Chemistry, Faculty of Science, Universiti Teknologi Malaysia, 81310 Skudai, Johor, Malaysia \\ ${ }^{2}$ Department of Chemistry, Faculty of Science, Universiti Putra Malaysia, 43400 Serdang, Selangor, Malaysia \\ ${ }^{3}$ Department of Polymer Engineering, Faculty of Chemical Engineering, Universiti Teknologi Malaysia, 81310 Skudai, Johor, Malaysia \\ ${ }^{4}$ School of Industrial Technology, Universiti Sains Malaysia, 11800 Minden, Penang, Malaysia \\ Correspondence should be addressed to Zainoha Zakaria; zainoha@kimia.fs.utm.my
}

Received 24 May 2013; Accepted 17 August 2013

Academic Editor: Gongnan Xie

Copyright (c) 2013 Zainoha Zakaria et al. This is an open access article distributed under the Creative Commons Attribution License, which permits unrestricted use, distribution, and reproduction in any medium, provided the original work is properly cited.

Poly (lactic acid) (PLA)/chitosan (CS) natural polymer/epoxidised natural rubber (ENR) composites were successfully prepared through a solution casting method. The morphological characteristics of fabricated composites were investigated by scanning electron microscopy (SEM) and optical microscopy. The microstructure of PLA/ENR was significantly altered with the addition of CS. SEM analysis of composites fractured surfaces revealed smooth and homogeneous texture and good dispersion of CS. However for $15 \mathrm{wt} \%$ CS composites, the phase segregation and poor adhesion between the polymers were observed. Fourier transform infrared spectroscopy revealed some levels of attractive interaction between CS, PLA, and ENR in the composites. The mechanical properties of composites in terms of tensile strength and tensile modulus were significantly improved with the addition of CS into the matrix while the percent elongation at break decreased. The tensile strength increased up to $5 \mathrm{wt} \% \mathrm{CS}$ loading for both PLA/CS and PLA/ENR/CS and thereafter decreased while Young's modulus increased up to $10 \mathrm{wt} \%$. However, when the CS content was increased to $15 \mathrm{wt} \%$, the tensile strength and tensile modulus were slightly decreased. These improvements were attributed to good dispersion of CS at the optimum filler levels and attractive interaction between the composites components.

\section{Introduction}

Nowadays, the use of natural polymers that are biodegradable and biocompatible has become increasingly important. This is due to their amazing characteristics: natural abundance, low costs, and wide range of applications $[1,2]$. These polymers are being widely used in the biomedical area, including wound dressing, drug delivery system, and tissue engineering scaffolds $[3,4]$. Polylactic acid (PLA) is prominent among the polymers that are biodegradable and biocompatible due to versatility of its applications and relatively low cost of production at industrial scale [5]. PLA is produced from renewable resources and has properties similar to most petroleumbased materials [6]. It has also been used in several applications such as food packaging, water and milk bottles, barriers for sanitary products, and diapers as well as in automotive applications [5, 7]. Nevertheless, it has few drawbacks which limit its use, including poor thermal stability, low water vapour and gas barrier properties, and embrittlement [8-10]. Blending of PLA with suitable synthetic or natural polymers could be a promising approach to overcome these inherent negative properties. In addition, blending of the polymer is an alternative way for preparation of biodegradable polymers having versatile properties such as good moisture-resistance and enhanced mechanical properties while maintaining their transparency and biodegradability [8]. Furthermore, blending is an easy and effective way to achieve multiphase polymeric materials with desired properties [11]. Solution blending is a convenient processing technique where a common solvent is used to dissolve the polymers.

Chitosan (poly-1, 4-D-glucosamine) is a renewable, natural, nontoxic, edible, and biodegradable polymer which is 
produced by alkaline deacetylation of chitin. It is one of the most abundant polymers that can be found in nature. Owing to its biodegradability and biocompatibility, CS is reported to be an active polymer with antimicrobial and antifungal activities [3, 11-14]. CS was used to create edible coatings or films to extend foodstuffs' shelf life, such as fruits, meat, fish, and seafood $[15,16]$. Furthermore, with growing concerns about environment, the development of new biodegradable packaging materials, such as CS films, could be an interesting alternative to petroleum-based synthetic polymers. In order to fulfill aforementioned applications, several studies have been devoted to produce new polymer systems incorporating CS as a bioactive material [17]. Thus CS could be an ideal candidate to blend with PLA.

However, the use of CS has been limited due to its poor mechanical properties, weak barrier properties of the film and high moisture sensitivity [18]. Again most of polymers pairs are, however, not miscible on a molecular scale. Immiscibility may result in phase-separated blends with a coarse morphology and poor mechanical properties. When polymers of very different polarities are blended, such as PLA and chitosan, it is necessary to use a third polymer or compatibilizer to reduce the interfacial tension between the polymers to decrease the particle size of the dispersed phase and to enhance the attractive interaction between the discrete phases so that compatible blend can be achieved. Epoxidised natural rubber (ENR) is nontoxic and biocompatible and has also been used as compatibilizer to enhance adhesion and compatibility between polymers. ENR is a suitable compatibilizer for PLA and CS because it forms compatible or miscible blends with CS [19].

A number of studies have been carried out on physical, mechanical, and morphological properties of PLA/CS blend [20, 21]. However, no single research work has been devoted to the blending of PLA/CS/ENR and its mechanical properties and morphological characterization. Motivated by earlier work [14], the current study was carried out to investigate the effect of CS on mechanical properties and morphological characteristics of PLA/CS and PLA/CS/ENR blend composites. Therefore, the aim of this study is to fabricate PLA/CS and PLA/CS/ENR composites through solution casting method to contrast their mechanical and morphological properties and to find the effect of CS loading on these properties of the resulting composites.

\section{Experimental}

2.1. Materials. Polylactic acid (NatureWork PLA 300ID) in pellet form was obtained from NatureWork LLC, Minnetonka, MN USA. It has a specific gravity $1.24 \mathrm{~g} / \mathrm{cm}^{3}$ and melt flow index (MFI) of $15 \mathrm{~g} / 10 \mathrm{~min}\left(190^{\circ} \mathrm{C} / 2.16 \mathrm{~kg}\right)$. Commercially available chitosan (practical grade $75 \%$ DD) from sigma Aldrich was used as the reinforcing filler. Epoxidised natural rubber (ENR) with 25 mol\% epoxidation (grade EPOXYPRENE 25) and specific gravity 0.97, used in this study, was used as received from the Malaysian Rubber Board, Malaysia. ENR-25 was used as compatibilizer. The solvent used was chloroform secured from Merck, Malaysia.

\subsection{Methods}

2.2.1. Preparation of PLA/CS and PLA/ENR/CS Blend Composites. A $10 \mathrm{wt} \%$ solution of PLA pellets in chloroform was prepared by stirring the solution inside the water bath at $60^{\circ} \mathrm{C}$, until the pellets were fully dissolved which is approximately 2 hours. The PLA solution was immediately cast on the clean glass plates and left to solvent evaporation at ambient temperature for 48 hours. The thickness of the cast solution was approximately $100 \mu \mathrm{m}$ and noted as neat PLA.

For the preparation of PLA/CS blend composites, different weights percentages (wt\%) of CS $(5,10$, and $15 \mathrm{wt} \%)$ were mixed into $100 \mathrm{~mL}$ chloroform, respectively, together with $10 \mathrm{~g}$ of PLA pellets; the solution was stirred inside the water bath at $60^{\circ} \mathrm{C}$, until the pellets were fully dissolved which is approximately 2 hours. The PLA/CS solution was immediately cast on the clean glass plates and left to solvent evaporate at ambient temperature for 48 hours. The thickness of the cast solution was approximately $100 \mu \mathrm{m}$ and noted as PLA/CS.

The PLA/CS/ENR blend composites were prepared by mixing the $10 \mathrm{wt} \%$ solution of PLA and $2 \mathrm{wt} \%$ of ENR with different chitosan loading $(5,10$, and $15 \mathrm{wt} \%)$ at $60^{\circ} \mathrm{C}$, with strong agitation until PLA pellets and ENR were dissolved and mixed with CS. The suspension was then sonicated for 2 minutes and was immediately cast on a clean glass plate to generate composite of about $100 \mu \mathrm{m}$ thick after removal of the solvent. The composites were designated as PLA/CS/ENR. Designation and formulations of PLA/ENR/CS composites are mentioned in Table 1.

2.2.2. Fourier Transforms Infrared Spectroscopy (FT-IR). FTIR was performed using a Perkin Elmer 1600 Infrared spectrometer. FT-IR spectra of the samples were recorded by using Nicolet's AVATAR 360 at 32 scans with a resolution of $4 \mathrm{~cm}^{-1}$ and within the wave number range of 4000 to $370 \mathrm{~cm}^{-1}$. The significant transmittance peak at a particular wave numbers was measured by using the "find peak tool" provided by Nicolet OMNIC 5.01 software.

2.2.3. Optical Microscopy. Optical microscopic technique was applied for the analysis of microstructure of the blend and composites samples. The magnified images of the samples were obtained using a microscope digital camera Leica DM 2500 M. Optical images of composites were taken at different points of the samples surfaces. All of the analyses were carried out at room temperature. The maximum magnification obtained with the optical microscope was about 100.

2.2.4. Scanning Electron Microscopy (SEM). The morphology of samples was observed by scanning electron microscopy (SEM). SEM analysis was carried out using a SEM-EDX Oxford INCA 400 model at an acceleration voltage $10 \mathrm{kV}$. The samples were sputter coated with gold to prevent charging. The micrographs were taken at a magnification of 1000 .

2.2.5. Mechanical Testing. Mechanical test was done using the Instron 4400 Universal Tester to measure the tensile strength at the point of breakage for each sample. Tensile tests were carried out at room temperature, according to 
TABLE 1: Designation and formulations of PLA/ENR/CS composites.

\begin{tabular}{lcccc}
\hline & PLA & CS (phr) & ENR (phr) & Designation \\
\hline PLA & 100 & - & - & PLA \\
\hline \multirow{3}{*}{ PLA/CS } & 100 & 5 & - & PLA 5CS \\
& 100 & 10 & - & PLA 10CS \\
& 100 & 15 & - & PLA 15CS \\
\hline PLA/ENR & 100 & - & 20 & P/ENR \\
\hline \multirow{3}{*}{ PLA/CS/ENR } & 100 & 5 & 20 & P/ENR 5CS \\
& 100 & 10 & 20 & P/ENR 10CS \\
& 100 & 15 & 20 & P/ENR 15CS \\
\hline
\end{tabular}

PLA: Polylactic acid; CS: chitosan; ENR: epoxidised natural rubber.

the ASTM D882 type V. A fixed crosshead rate of $10 \mathrm{~mm} / \mathrm{min}$ was utilized in all cases and the results were taken as an average of five tests.

\section{Results and Discussion}

3.1. Fourier Transform Infrared Spectroscopy (FT-IR). FT-IR is a well-known and widely used method to investigate the intermolecular interaction and phase behavior between the polymers. In this study, the interaction between PLA, CS, and ENR was investigated by FT-IR spectroscopy and is shown in Figure 1. The FTIR spectrum of the neat PLA (Figure 1(a)) clearly showed the characteristic absorption band in the region of $3500-3600 \mathrm{~cm}^{-1}, 2946-2999 \mathrm{~cm}^{-1}$, and $1757 \mathrm{~cm}^{-1}$ due to $\mathrm{O}-\mathrm{H}$ bending and stretching vibration, $\mathrm{C}-\mathrm{H}$ asymmetric stretching vibration, and $\mathrm{C}=\mathrm{O}$ stretching vibration, respectively $[22,23]$. The FTIR spectra of PLA also showed the presence of bands at $1448 \mathrm{~cm}^{-1}$ for $\mathrm{C}-\mathrm{H}$ stretching in the $\mathrm{CH}_{3}$ and $957 \mathrm{~cm}^{-1}$ and $867 \mathrm{~cm}^{-1}$ for C$\mathrm{C}$ single bond [24]. On the contrary, it can be seen from Figure 1(b) that the characteristic absorption band of PLA at $3418 \mathrm{~cm}^{-1}$ and $2918 \mathrm{~cm}^{-1}$, which is due to $\mathrm{O}-\mathrm{H}$ and $\mathrm{C}=\mathrm{O}$ stretching vibration, has shifted towards lower wave numbers with narrowband intensity upon incorporation of CS. It was also found that the prominent band intensity at 2946$2999 \mathrm{~cm}^{-1}$ and $3504 \mathrm{~m}^{-1}$ has significantly decreased by the addition of CS. Therefore, it can be implied that CS adequately dispersed in the PLA matrix with some levels of interaction between them forming the PLA-CS composites. Similar result has also been reported by other researchers [25].

Figures 1(c) and 1(d) represented the FT-IR spectra of PLA/ENR and PLA/CS/ENR composites, respectively. It was found that the characteristic absorption band of PLA/ENR in the region of $3500-3600 \mathrm{~cm}^{-1}$ and $1757 \mathrm{~cm}^{-1}$ due to hydroxyl $(\mathrm{O}-\mathrm{H})$ and $\mathrm{C}=\mathrm{O}$ vibration has shifted towards lower wave numbers when CS was added into PLA/ENR matrix. This blue shift indicated some level of interaction and the formation of PLA/CS/ENR composites. It is also interesting to note that the relatively broad band intensity peaks at 3500 to $3600 \mathrm{~cm}^{-1}$ and $1757 \mathrm{~cm}^{-1}$ was considerable reduced and partially disappeared $\left(3405 \mathrm{~cm}^{-1}\right)$ in PLA/CS/ENR composites. All these pointed towards good dispersion and interaction between PLA, ENR, and CS, which significantly changed the morphological characteristics of the composites.

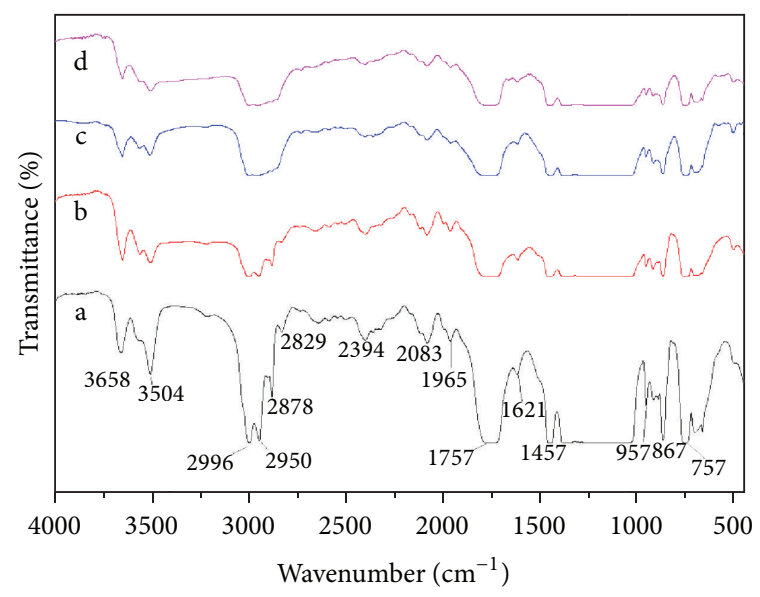

FIGURE 1: FT-IR results of (a) PLA, (b) PLA/CS, (c) PLA/ENR, and (d) PLA/ENR/CS.

3.2. Optical Microstructure. In order to observe the CS dispersion on PLA and PLA/ENR composites, surfaces were characterized by optical microscopy (OM). Figures 2(a) to 2(d) show the typical optical microstructures of pure PLA, PLA/ENR, PLA/CS, and PLA/CS/ENR blend composites. From Figure 2(a), it can be seen that the microstructure of pure PLA seems to display smooth surface as compared with PLA/ENR (Figure 2(b)). There is a clear phase separation observed from the microstructure of PLA/ENR composites. This is may be due to the incompatibility between PLA and ENR and also the poor interfacial interaction between two polymers [26].

Meanwhile, for the PLA/CS and PLA/CS/ENR composites (Figures 2(c) and 2(d)), white spots were observed on the surfaces of the composites. These white spots are indicating the CS particles. From Figures 2(c) and 2(d), it is clearly observed that CS particles are not uniformly dispersed within the matrix and the aggregation of CS on the surface of composite was readily apparent. The composites of PLA/CS/ ENR show a rough surface morphology with number of microvoids throughout the composite surfaces as compared with the PLA/CS. Similar results have also been reported by other researchers [27].

3.3. Scanning Electron Microscopic (SEM) Analysis. In order to obtain a clear insight into the surface morphology, scanning electron microscopic (SEM) analysis was attempted. SEM is known to be the best choice because of its potential in precise analysis of a solid surface. The fractured surfaces of the composites used in tensile tests were observed by SEM. SEM micrographs of different fractured composite samples are shown in Figures 3(a) to 3(d). Figure 3(a) shows that the PLA fractured surfaces were covered with uneven fibrils, layers, and a number of microvoids throughout the surface. Therefore, the fractured surface of pure PLA could be categorized as ductile fracture [15]. This is because the fibrils remained on the fracture surface. Additionally, fractures producing fibrils less than $1 \mu \mathrm{m}$ long are categorized in brittle fracture [22]. 


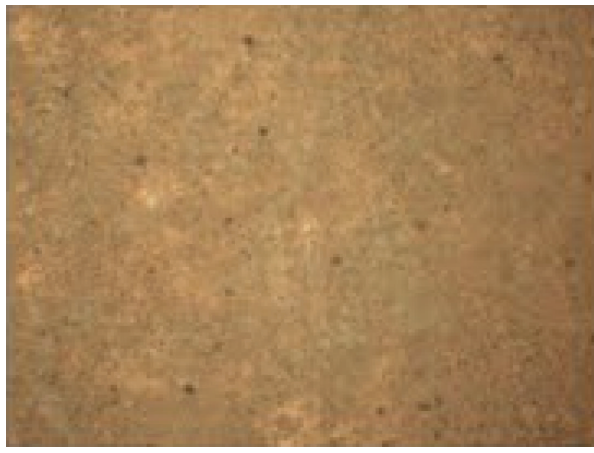

(a)

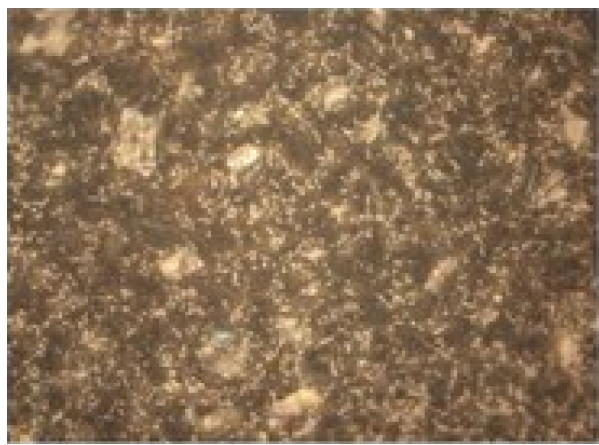

(c)

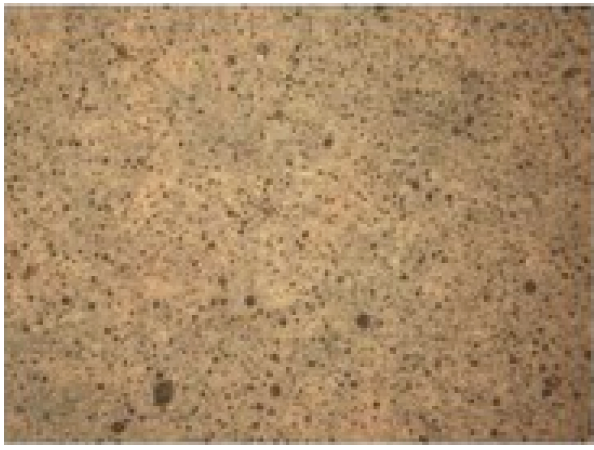

(b)

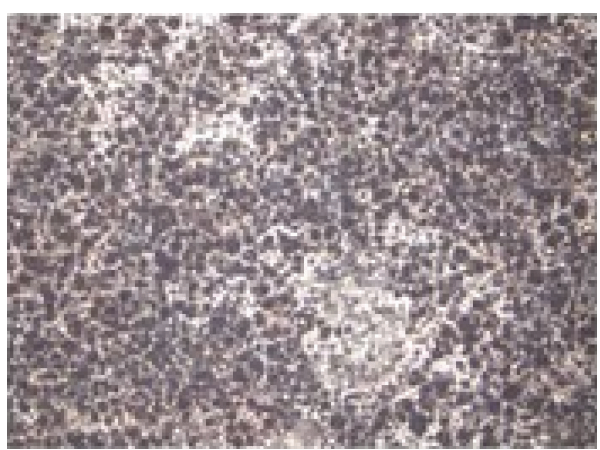

(d)

Figure 2: Optical microstructures of (a) PLA, (b) PLA/ENR, (c) PLA/CS, and (d) PLA/CS/ENR.

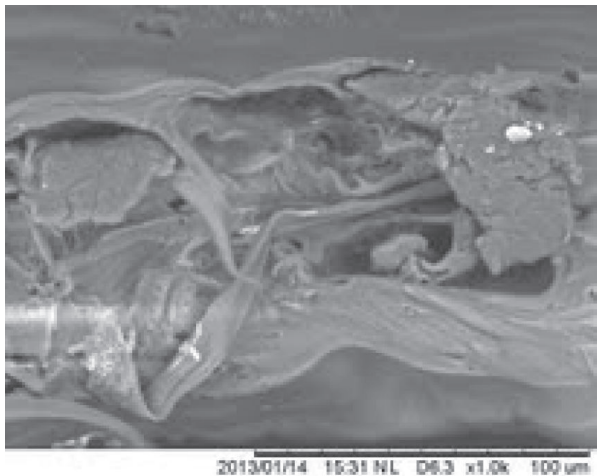

(a)

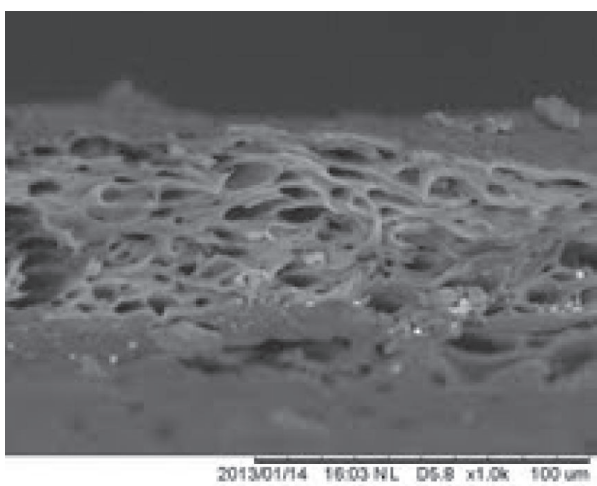

(c)

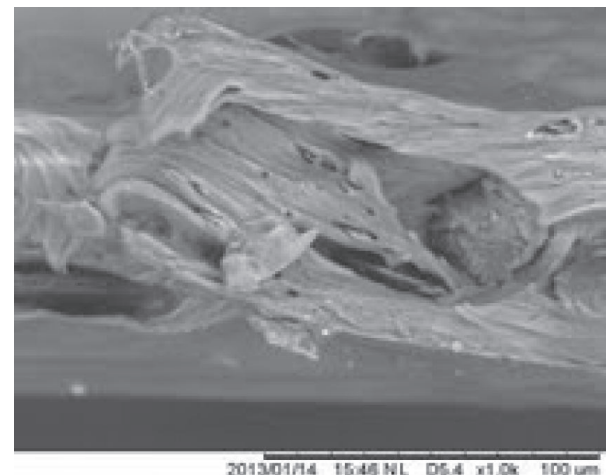

(b)

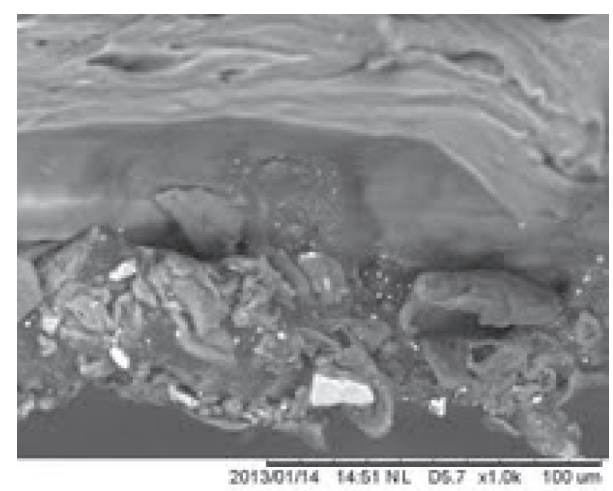

(d)

Figure 3: Typical SEM of (a) PLA, (b) PLA/CS (10\% CS), (c) PLA/ENR, and (d) PLA/CS/ENR (10\% CS). 
Conversely, the tensile test fractured surfaces of PLA/CS samples (Figure 3(b)) showed smooth and homogeneous surfaces upon incorporation of CS in the PLA matrix. It can also be seen that the fibrils and microholes significantly reduced after addition of CS into the PLA matrix. Furthermore, the fractured surface of the PLA/CS seems to have no interface layer and more homogeneous compared with pure PLA. This result indicates that the PLA and CS were well blended in the matrix and the interaction between PLA and CS was strong. Thus, this result confirms that PLA and CS at this concentration are compatible. However, at higher loading (above 10\%) of CS, the SEM image shows agglomeration and poor distribution of the excess CS in the PLA matrix. This demonstrated that beyond the optimum concentration CS tends to agglomerate presumably due to van der Waals forces leading to a fiber bundle formation which also results in a poor interfacial adhesion between PLA and CS in the case of higher CS content in the PLA/CS composites. Consequently, lower mechanical properties are displayed for the higher content of CS in the PLA matrix. Similar result was also reported by other researchers $[15,18]$.

Figure 3(c) shows the fractured surfaces of PLA/ENR composites. From the figure, it can be observed that the two polymers (PLA and ENR) were not miscible. There are widespread microvoids existing on the composites fractured surface. Moreover, the clear gaps were observed between PLA and ENR polymer matrixes. This indicates that the adhesion and interaction between PLA and ENR are poor. Therefore, the mechanical strength of PLA/ENR composites was found to be lower as compared with pure PLA. By adding CS particles into PLA/ENR matrix, morphological properties of composites are expected to be improved.

The miscibility increased significantly when the CS was incorporated in the blend. The miscibility between the polymers was found to increase with increase in the amount of CS loading up to $10 \%$. Furthermore, the fractured surface of the PLA/ENR became homogenous on addition of CS. This might be due to the improvement in compatibility between PLA, CS, and ENR. This result also indicates the adequate and uniform dispersion of CS in the polymer matrix, by increasing the interfacial adhesion between PLA and ENR. However, the surface appeared little bit rough on addition of $15 \%$ CS. Agglomeration of excess CS above the optimum 10\% might be responsible for this observation.

3.4. Mechanical Properties. Tensile properties of PLA/CS and PLA/CS/ENR composites were investigated in order to find the effect of CS loading. A typical stress-strain curve for 5\% CS filled PLA composite is shown in Figure 4. The tensile strength, tensile modulus, and percentage elongation at break of PLA/CS and PLA/CS/ENR composites at different percentages of CS loading are mentioned in Table 2 and shown in Figures 5 to 7 , respectively. It can be seen from Figures 5 and 6 that in general both tensile strength and tensile modulus of composites increased on addition of CS as reinforcement filler. CS acted as reinforcement filler and attractively interacted with the different polymers resulting in improved tensile strength and tensile modulus.

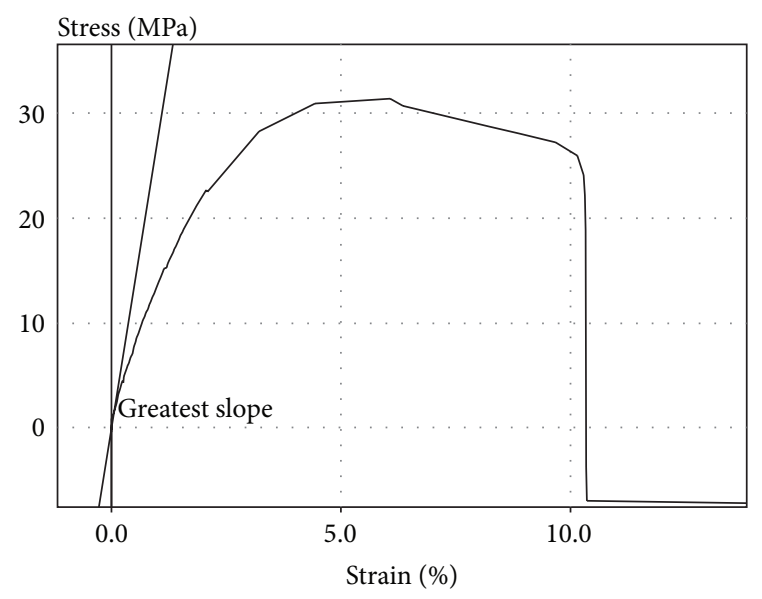

FIgURE 4: A typical stress-strain curve for 5\% CS filled PLA composite.

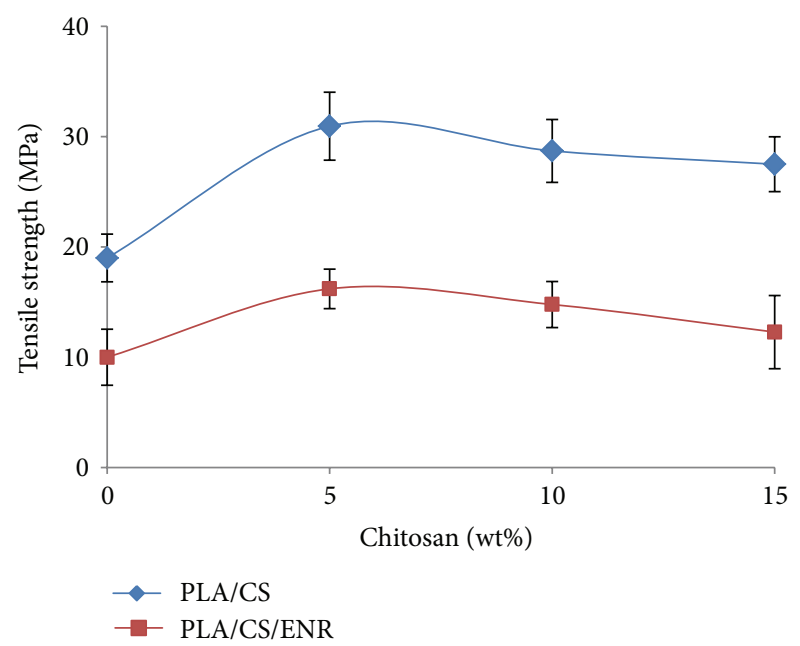

FIGURE 5: Variation of tensile strength at different CS loading showing possible data trend.

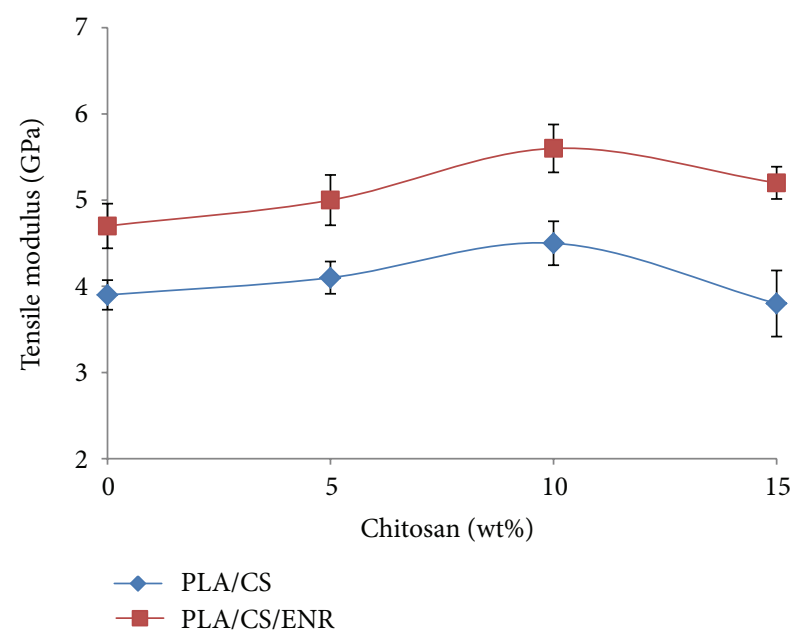

FIGURE 6: Variation of tensile modulus at different CS loading showing possible data trend. 
TABLE 2: Tensile properties of PLA/ENR/CS composites.

\begin{tabular}{lccc}
\hline & $\begin{array}{c}\text { Tensile strength } \\
(\mathrm{MPa})\end{array}$ & $\begin{array}{c}\text { Tensile modulus } \\
(\mathrm{MPa})\end{array}$ & $\begin{array}{c}\text { Percentage } \\
\text { strain } \\
\text { at break }\end{array}$ \\
\hline PLA & 19.00 & 3.90 & - \\
PLA 5CS & 30.95 & 4.10 & 6.86 \\
PLA 10CS & 28.71 & 4.50 & 5.22 \\
PLA 15CS & 27.50 & 3.80 & 4.98 \\
PLA/ENR & 10.00 & 4.70 & - \\
P/ENR 5CS & 16.20 & 5.00 & 12.16 \\
P/ENR 10CS & 14.78 & 5.60 & 8.40 \\
P/ENR 15CS & 12.28 & 5.20 & 6.10 \\
\hline
\end{tabular}

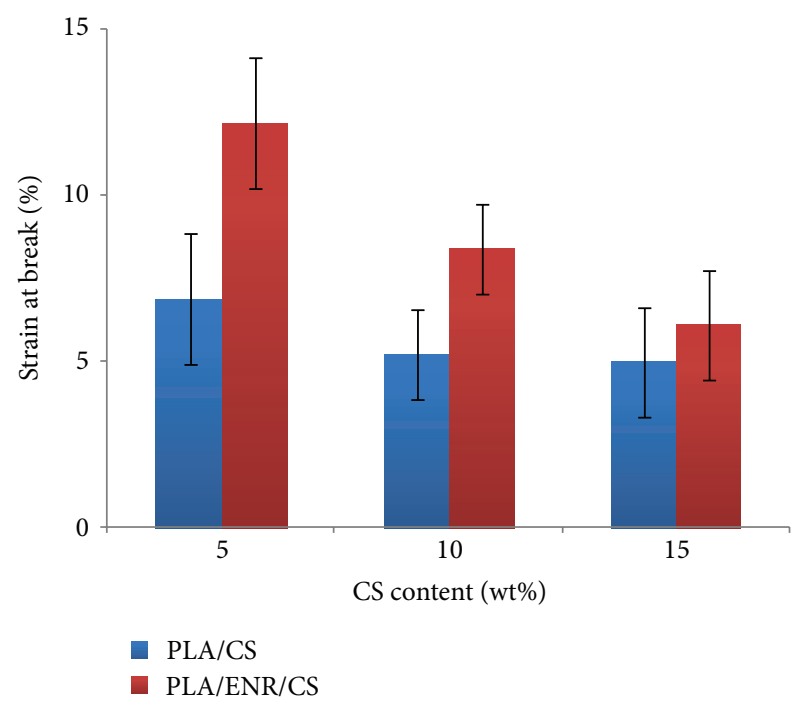

Figure 7: Variation of elongation at break at different CS loading showing possible data trend.

Figure 5 shows tensile strength of the PLA/CS and PLA/ CS/ENR composites. Tensile strength increased with increase in CS loading up to $5 \mathrm{wt} \%$ and then decreased gradually up to $15 \mathrm{wt} \%$. This implies that $5 \mathrm{wt} \%$ is the optimum concentration beyond which agglomeration of CS particles occur causing stress concentration within the matrix which consequently lowers the tensile strength. This claim is supported by result of SEM and optical microstructural analysis in which congestion and agglomeration of CS particles in the polymer matrix are observed. Furthermore, a weak interface surrounding the aggregated CS particles may also have caused the decrease in tensile strength.

The tensile modulus of PLA/CS and PLA/CS/ENR composites are compared in Figure 6. Based on this figure it is clear that the PLA/CS exhibited a lower tensile modulus than the PLA/CS/ENR at all CS loading. Increase in tensile modulus with CS loading is a typical behavior of polymer filled systems [18]. During tensile loading, partially separated microspaces are created, which hinder stress propagation between the CS and polymer matrixes [28]. The degree of obstruction increases with increase in CS loading. This consequently increases the stiffness. However, at $15 \mathrm{wt} \%$, the tensile modulus of PLA/CS and PLA/CS/ENR decreased slightly. Nonetheless the tensile modulus of PLA/CS/ENR was $10.6 \%$ higher than that of PLA/ENR. This may be due to the incorporation of CS into the PLA matrix, which increased the compatibility and adhesion between PLA, CS, and ENR, thereby resulting in higher tensile strength for composites.

Figure 7 shows the results of percent elongation at break for PLA/CS and PLA/CS/ENR. It can be seen that the elongation at break of the composite decreased gradually as the CS content is increased for both PLA/CS and PLA/CS/ENR. These observations may be attributed to the stiffening action of the filler by restricting the segmental chain movement of PLA during tensile testing. According to Pei and Berglund, the elongation at break is affected by the volume fraction of the added reinforcement, the dispersion of the reinforcement in the matrix, and the interaction between the reinforcement and the matrix [29]. The figure also shows that the elongation at break decreased steadily with increasing CS content for both PLA/ENR and PLA/CS/ENR in comparison with the pure PLA which exhibited $97.8 \pm 0.5 \%$ of elongation at break. It is however interestingly to note that \% elongation at break of PLA/CS/ENR composites showed higher value compared with PLA/CS. These observations may be due to the elastomeric nature of ENR. The restricting effect of CS may have caused substantial local stress concentrations and reduced the elongation at break [29]. This assertion is supported by the optical microscopy and SEM results in which agglomeration of CS particles is clearly observed.

\section{Conclusions}

The following conclusions are drawn from the experimental work.

(1) Microstructural studies showed good attractive interaction among the composites' components with visible agglomeration at higher CS loading (15 wt\%).

(2) Tensile strength for both composites increased up to $5 \mathrm{wt} \%$ CS loading while tensile modulus showed improvements up to $10 \mathrm{wt} \%$.

(3) Percent elongation at break decreased steadily with increasing CS loading which is attributed to chain movement restriction caused by the stiffer CS particles.

(4) Improvements in the composites properties could be attributed to better interphase attractive interaction and adhesion of the incorporated CS particles in the polymer matrix.

(5) FT-IR analysis indicated some level of attractive interaction between CS, ENR, and PLA. From the previously mentioned, it is hereby proposed that CS can be considered as reinforcing filler for the PLA/CS and PLA/CS/ENR systems.

(6) The ENR served as a compatibilizer for the PLA/CS by enhancing their attractive interaction leading to the observed improvements in the properties of the composites. 


\section{Conflict of Interests}

The authors would like to inform that there is no conflict of interests with any trademark mentioned in the paper.

\section{Acknowledgment}

The authors would like to thank the Research University Grant 05H22 subcode Q.J130000.2509.05H22 for financial support.

\section{References}

[1] D. Li and Y. Xia, "Electrospinning of nanofibers: reinventing the wheel?” Advanced Materials, vol. 16, no. 14, pp. 1151-1170, 2004.

[2] M. K. M. Haafiz, S. J. Eichhorn, A. Hassan, and M. Jawaid, "Isolation and characterization of microcrystalline cellulose from oil palm biomass residue," Carbohydrate Polymers, vol. 93, pp. 628-634, 2013.

[3] N. M. Julkapli, H. M. Akil, and Z. Ahmad, "Preparation, properties and applications of chitosan-based biocomposites/blend materials: a review," Composite Interfaces, vol. 18, no. 6, pp. 449507, 2011.

[4] A. Domard, "A perspective on 30 years research on chitin and chitosan," Carbohydrate Polymers, vol. 84, no. 2, pp. 696-703, 2011.

[5] M. Jonoobi, J. Harun, A. P. Mathew, and K. Oksman, "Mechanical properties of cellulose nanofiber (CNF) reinforced polylactic acid (PLA) prepared by twin screw extrusion," Composites Science and Technology, vol. 70, no. 12, pp. 1742-1747, 2010.

[6] R. E. Drumright, P. R. Gruber, and D. E. Henton, "Polylactic acid technology," Advanced Materials, vol. 12, pp. 1841-1846, 2000.

[7] A. P. Mathew, K. Oksman, and M. Sain, "Mechanical properties of biodegradable composites from poly lactic acid (PLA) and microcrystalline cellulose (MCC)," Journal of Applied Polymer Science, vol. 97, no. 5, pp. 2014-2025, 2005.

[8] K. Oksman, A. P. Mathew, D. Bondeson, and I. Kvien, "Manufacturing process of cellulose whiskers/polylactic acid nanocomposites," Composites Science and Technology, vol. 66, no. 15, pp. 2776-2784, 2006.

[9] L. Petersson, I. Kvien, and K. Oksman, "Structure and thermal properties of poly(lactic acid)/cellulose whiskers nanocomposite materials," Composites Science and Technology, vol. 67, no. 1112, pp. 2535-2544, 2007.

[10] P. Qu, Y. Gao, G.-F. Wu, and L.-P. Zhang, "Nanocomposites of Poly(lactic acid) reinforced with cellulose nanofibrils," Bioresources, vol. 5, no. 3, pp. 1811-1823, 2010.

[11] T. Tanabe, N. Okitsu, A. Tachibana, and K. Yamauchi, "Preparation and characterization of keratin-chitosan composite film," Biomaterials, vol. 23, no. 3, pp. 817-825, 2002.

[12] R. A. A. Muzzarelli, "Chitins and chitosans for the repair of wounded skin, nerve, cartilage and bone," Carbohydrate Polymers, vol. 76, no. 2, pp. 167-182, 2009.

[13] M. N. V. R. Kumar, R. A. A. Muzzarelli, C. Muzzarelli, H. Sashiwa, and A. J. Domb, "Chitosan chemistry and pharmaceutical perspectives," Chemical Reviews, vol. 104, no. 12, pp. 6017-6084, 2004.

[14] Z. Zakaria, Z. Izzah, M. Jwaid, and A. Hassan, "Effect of degree of deacetylation chitosan on theremal stability and compatibility of chitosan polyamid blend," Bioresources, vol. 7, pp. 55685580, 2012.
[15] D. Jeevitha and K. Amarnath, "Chitosan/PLA nanoparticles as a novel carrier for the delivery of anthraquinone: synthesis, characterization and in vitro cytotoxicity evaluation," Colloids and Surfaces B, vol. 101, pp. 126-134, 2013.

[16] R. Jayakumar, M. Prabaharan, S. V. Nair, and H. Tamura, "Novel chitin and chitosan nanofibers in biomedical applications," Biotechnology Advances, vol. 28, no. 1, pp. 142-150, 2010.

[17] H. Tamura, T. Furuike, S. V. Nair, and R. Jayakumar, "Biomedical applications of chitin hydrogel membranes and scaffolds," Carbohydrate Polymers, vol. 84, no. 2, pp. 820-824, 2011.

[18] N. E. Suyatma, A. Copinet, V. Coma, and F. Fricoteaux, "Compatibilization method applied to the chitosan-acid poly $(\mathrm{L}-$ lactide) solution," Journal of Applied Polymer Science, vol. 117, no. 5, pp. 3083-3091, 2010.

[19] H. Balakrishnan, N. Nematzadeh, M. U. Wahit, H. Hassan, and M. Imran, "Epoxidized natural rubber toughened polyamide 6/ organically modified montmorillonite nanocomposites," Journal of Thermoplastic Composite Materials, vol. 26, pp. 1-18, 2012.

[20] C. Chen, L. Dong, and M. K. Cheung, "Preparation and characterization of biodegradable poly(l-lactide)/chitosan blends," European Polymer Journal, vol. 41, no. 5, pp. 958-966, 2005.

[21] M. Peesan, P. Supaphol, and R. Rujiravanit, "Preparation and characterization of hexanoyl chitosan/polylactide blend films," Carbohydrate Polymers, vol. 60, no. 3, pp. 343-350, 2005.

[22] G. H. Yew, M. A. M. Yusof, M. Z. A. Ishak, and U. S. Ishiaku, "Water absorption and enzymatic degradation of poly(lactic acid)/rice starch composites," Polymer Degradation and Stability, vol. 90, no. 3, pp. 488-500, 2005.

[23] Q. Cheng, S. Wang, and T. G. Rials, "Poly(vinyl alcohol) nanocomposites reinforced with cellulose fibrils isolated by high intensity ultrasonication," Composites Part A, vol. 40, no. 2, pp. 218-224, 2009.

[24] S. J. Eichhorn, A. Dufresne, M. Aranguren et al., "Current international research into cellulose nanofibres and nanocomposites," Journal of Materials Science, vol. 45, no. 1, pp. 1-33, 2010.

[25] D. Li and Y. Xia, "Electrospinning of nanofibers: reinventing the wheel?" Advanced Materials, vol. 16, no. 14, pp. 1151-1170, 2004.

[26] V. M. Correlo, L. F. Boesel, M. Bhattacharya, J. F. Mano, N. M. Neves, and R. L. Reis, "Properties of melt processed chitosan and aliphatic polyester blends," Materials Science and Engineering A, vol. 403, no. 1-2, pp. 57-68, 2005.

[27] V. M. Correlo, L. F. Boesel, M. Bhattacharya, J. F. Mano, N. M. Neves, and R. L. Reis, "Hydroxyapatite reinforced chitosan and polyester blends for biomedical applications," Macromolecular Materials and Engineering, vol. 290, no. 12, pp. 1157-1165, 2005.

[28] R. Grande and A. J. F. Carvalho, "Compatible ternary blends of chitosan/poly(vinyl alcohol)/poly(lactic acid) produced by oilin-water emulsion processing," Biomacromolecules, vol. 12, no. 4, pp. 907-914, 2011.

[29] A. Pei, Q. Zhou, and L. A. Berglund, "Functionalized cellulose nanocrystals as biobased nucleation agents in poly(l-lactide) (PLLA)-crystallization and mechanical property effects," Composites Science and Technology, vol. 70, no. 5, pp. 815-821, 2010. 

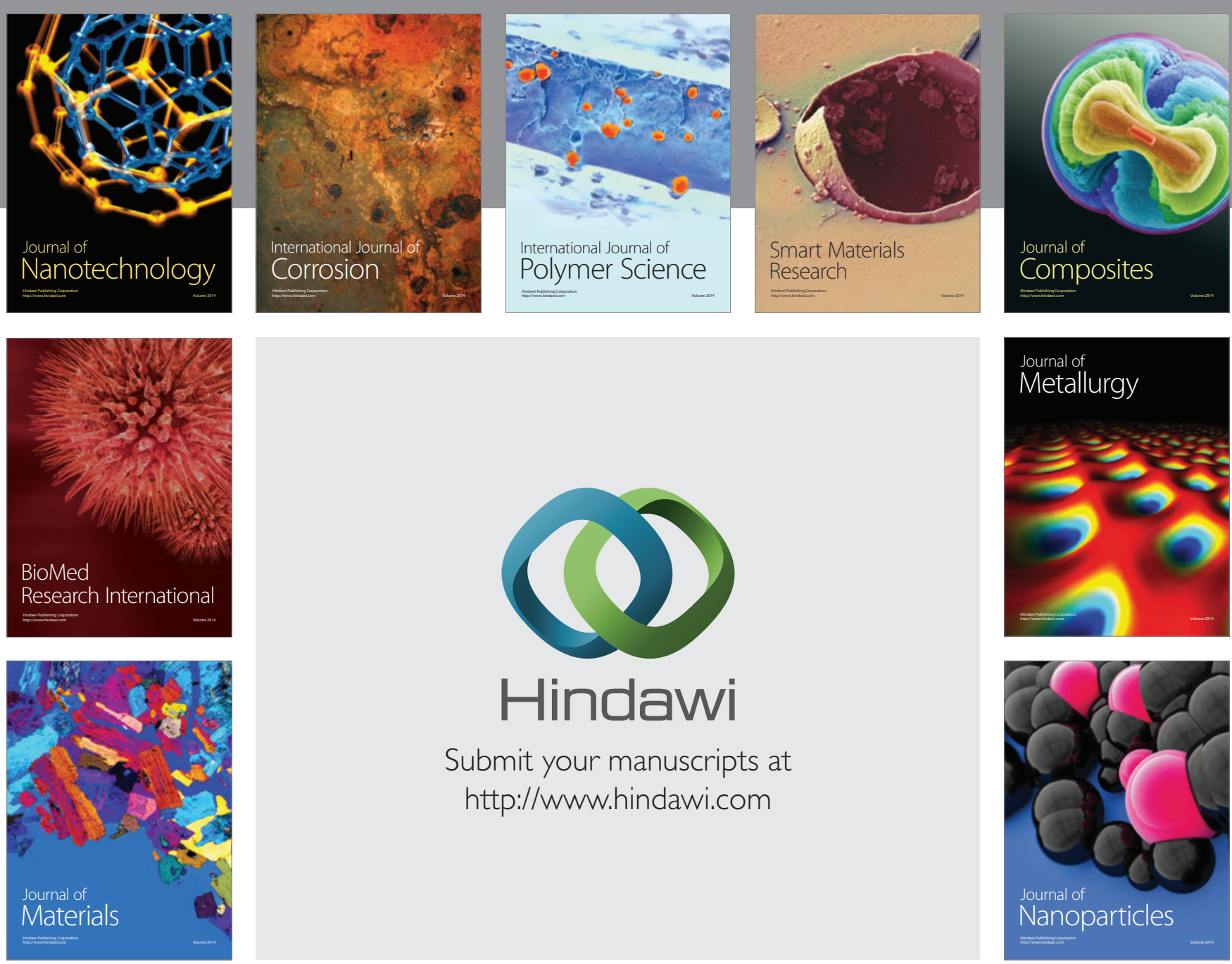

Submit your manuscripts at http://www.hindawi.com
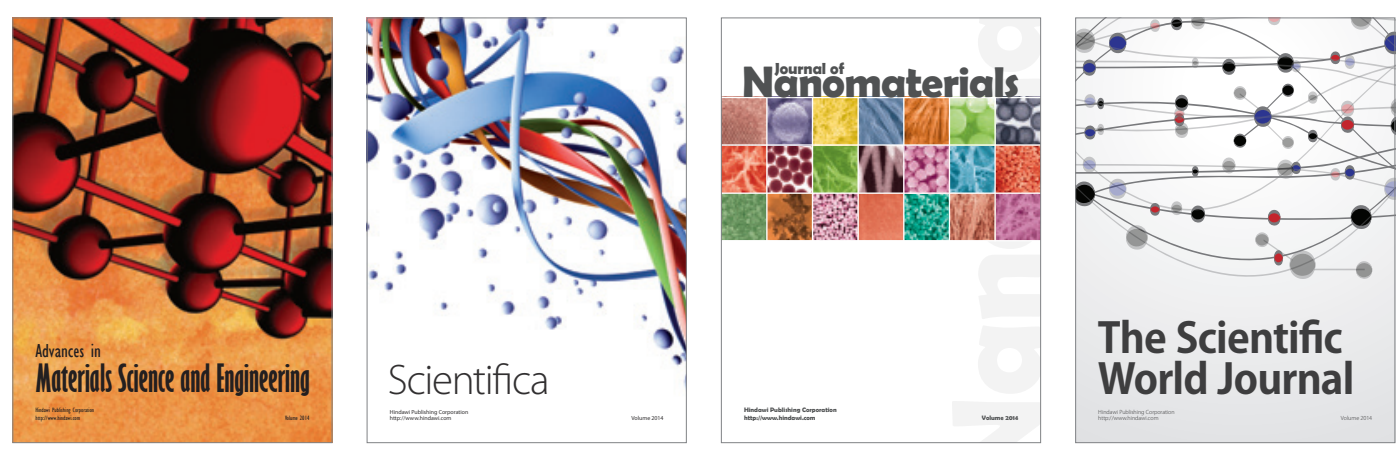

\section{The Scientific World Journal}
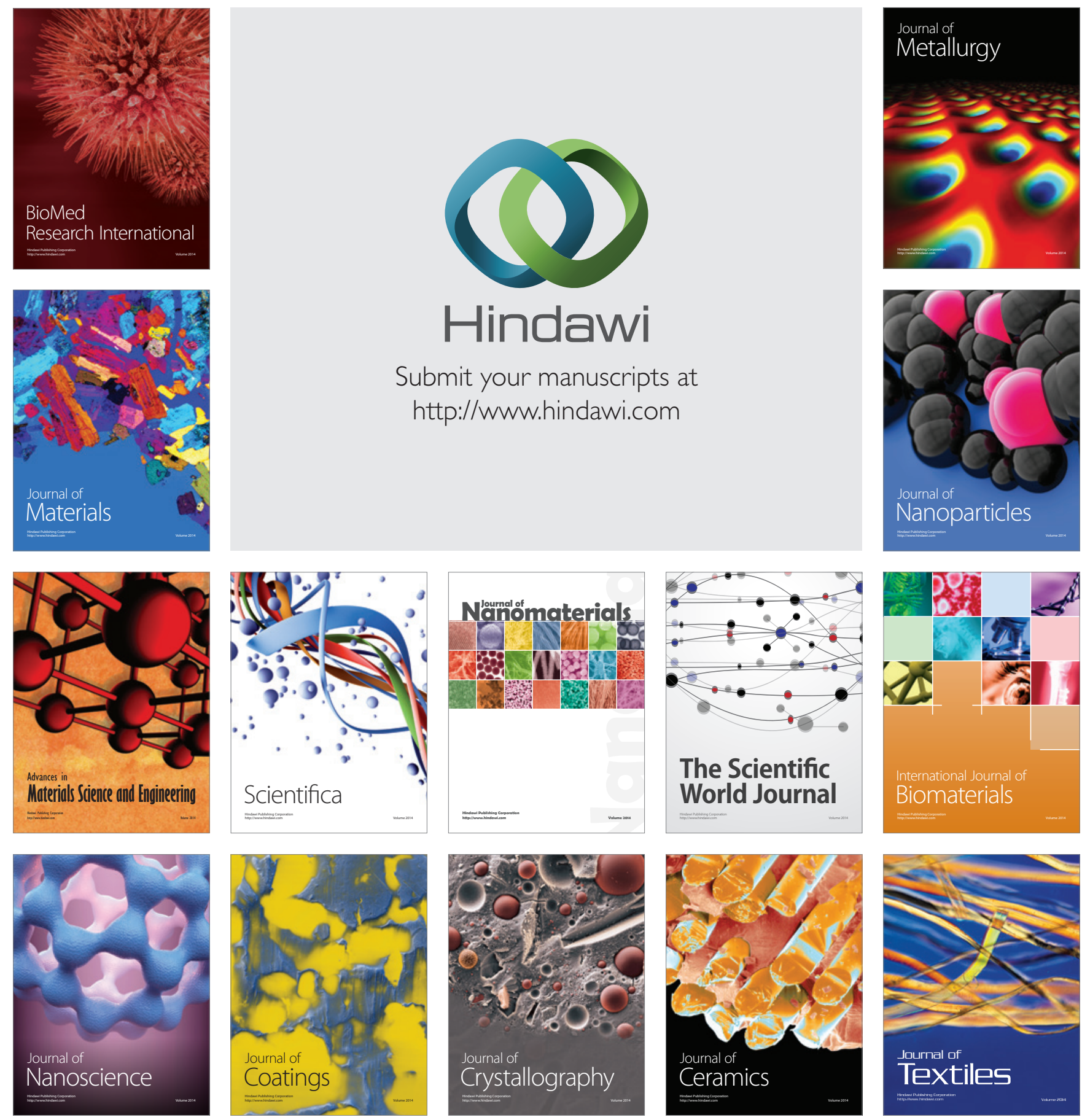